PROCEEDINGS OF THE

AMERICAN MATHEMATICAL SOCIETY

Volume 133, Number 4, Pages 995-997

S 0002-9939(04)07755-X

Article electronically published on October 14, 2004

\title{
RELATING EXPONENTIAL GROWTH IN A MANIFOLD AND ITS FUNDAMENTAL GROUP
}

\author{
ANTHONY MANNING
}

(Communicated by Michael Handel)

\begin{abstract}
We relate the growth rate of volume in the universal cover of a compact Riemannian manifold to the growth in the fundamental group in terms of word length in a given set of generators and the length of geodesics representing these generators.
\end{abstract}

Given a group $\Gamma$ and a finite set $S$ of generators for $\Gamma$, we have the word length $l_{S}: \Gamma \rightarrow \mathbb{N} \cup\{0\}$ for which $l_{S}(\gamma)$ is the least $n$ such that there exist $s_{1}, \ldots, s_{n} \in$ $S \cup S^{-1}$ with $\gamma=s_{1} \ldots s_{n}$. The exponential growth rate of $\Gamma$ is defined as $\psi(\Gamma, S):=$ $\lim _{k \rightarrow \infty} k^{-1} \log \#\left\{\gamma \in \Gamma: l_{S}(\gamma) \leq k\right\}$. It is easy to check that $\psi(\Gamma, S)>0$ if and only if $\psi\left(\Gamma, S^{\prime}\right)>0$ for any finite set $S^{\prime}$ of generators of $\Gamma$; in this case $\Gamma$ is said to be of exponential growth. Without a way of comparing the length of elements of different sets $S$ of generators there is no single natural value of the exponential growth rate of $\Gamma$.

When $\Gamma$ is the fundamental group $\pi_{1}(M, *)$ of a compact Riemannian manifold $(M, g)$ we can define $L_{g}: \Gamma \rightarrow \mathbb{R}$ by putting $L_{g}(\gamma)$ equal to the shortest length of a geodesic from the base point $*$ to itself representing $\gamma$. In the Cayley graph (see [3], for example) of $(\Gamma, S)$ we shall use $L_{g} \mid S$ instead of 1 for the lengths of edges and incorporate this length into a definition of the growth rate of $\Gamma$ for the generating set $S$. Thus we define $L_{g, S}: \Gamma \rightarrow \mathbb{R}$ by

$$
L_{g, S}(\gamma):=\inf \left\{\sum_{j=1}^{n} L_{g}\left(s_{j}\right): \gamma=s_{1} \ldots s_{n},\left\{s_{1}, \ldots, s_{n}\right\} \subset S \cup S^{-1}, n \in \mathbb{N} \cup\{0\}\right\} .
$$

(Note that this infimum is attained and that $L_{g, S}\left|S=L_{g}\right| S$.) This gives rise to the growth function

$$
\beta_{g, S}: \mathbb{R} \rightarrow \mathbb{R}, \beta_{g, S}(t):=\#\left\{\gamma \in \Gamma: L_{g, S}(\gamma) \leq t\right\}
$$

and the exponential growth rate

$$
\varphi(g, S):=\lim _{t \rightarrow \infty} t^{-1} \log \beta_{g, S}(t) .
$$

This limit exists because $\beta_{g, S}(t+u) \leq \beta_{g, S}(t) \beta_{g, S}(u)$. Thus $\varphi(g, S)$ differs from $\psi(\Gamma, S)$ by incorporating the $g$-length of geodesics representing the generators in $S$.

Received by the editors December 10, 2003.

2000 Mathematics Subject Classification. Primary 20F69, 37D40; Secondary 20F65, 37B40.

(C)2004 American Mathematical Society Reverts to public domain 28 years from publication 
The volume entropy of $(M, g)$ is defined by

$$
h(g):=\lim _{R \rightarrow \infty} R^{-1} \log \operatorname{Vol} B(*, R),
$$

the growth rate of the $\widetilde{g}$-volume of the ball of radius $R$ and centre the base point $*$ in the universal cover $(\widetilde{M}, \widetilde{g})$ of $(M, g)$. See [6] or [5, 8] for this and its connection with the topological entropy of the geodesic flow. By tiling $\widetilde{M}$ with the translates of a fundamental domain by the elements of the covering group $\Gamma$ we see that

$$
h(g)=\lim _{R \rightarrow \infty} R^{-1} \log \#\left\{\gamma \in \Gamma: L_{g}(\gamma) \leq R\right\} .
$$

Our theorem connects the volume entropy with the exponential growth rate of $\Gamma$ for generating subsets $S \subset \Gamma$.

\section{Theorem 1.}

$$
h(g)=\sup \left\{\varphi(g, S): S \text { is a finite subset generating } \Gamma=\pi_{1}(M, *)\right\} .
$$

Proof. First we fix a finite generating set $S \subset \Gamma$ and argue that $\varphi(g, S) \leq h(g)$. For $\gamma \in \Gamma, L_{g, S}(\gamma)$ is the infimum of the length of certain piecewise geodesic loops representing $\gamma$, and so $L_{g}(\gamma) \leq L_{g, S}(\gamma)$. Thus

$$
\#\left\{\gamma \in \Gamma: L_{g, S}(\gamma) \leq R\right\} \leq \#\left\{\gamma \in \Gamma: L_{g}(\gamma) \leq R\right\},
$$

from which we obtain $\varphi(g, S) \leq h(g)$.

Choose a fundamental domain $N$ for $\widetilde{M}$ of diameter $A$, say, using the metric $d$ on $\widetilde{M}$ arising from the Riemannian metric $\widetilde{g}$. Consider the fibre $\{\alpha *: \alpha \in \Gamma\}$ over the base point $* \in M$. Given $\gamma \in \Gamma$ with $L_{g}(\gamma) \leq k R$, we pick $\alpha_{j} \in \Gamma, 1 \leq j<k$, such that $d\left(\alpha_{j} *, \gamma(j R)\right) \leq A$ and put $\alpha_{0}=\operatorname{id}_{\Gamma}, \alpha_{k}=\gamma$. Then, for $1 \leq j<k$,

$$
\begin{aligned}
L_{g}\left(\alpha_{j}^{-1} \alpha_{j+1}\right) & =d\left(\alpha_{j} *, \alpha_{j+1} *\right) \\
& \leq d\left(\alpha_{j} *, \gamma(j R)\right)+d(\gamma(j R), \gamma((j+1) R))+d\left(\gamma((j+1) R), \alpha_{j+1} *\right) \\
& \leq R+2 A .
\end{aligned}
$$

Put

$$
S:=\left\{\alpha \in \Gamma: L_{g}(\alpha) \leq R+2 A\right\}
$$

Then

$$
L_{g, S}(\gamma)=L_{g, S}\left(\alpha_{1}\left(\alpha_{1}^{-1} \alpha_{2}\right)\left(\alpha_{2}^{-1} \alpha_{3}\right) \ldots\left(\alpha_{k-1}^{-1} \gamma\right)\right) \leq k(R+2 A) .
$$

So $\#\left\{\gamma \in \Gamma: L_{g}(\gamma) \leq k R\right\} \leq \beta_{g, S}(k(R+2 A))$. Thus

$$
\begin{aligned}
h(g) & =\lim _{k \rightarrow \infty}(k R)^{-1} \log \#\left\{\gamma \in \Gamma: L_{g}(\gamma) \leq k R\right\} \\
& \leq \frac{k(R+2 A)}{k R} \lim _{k \rightarrow \infty}(k(R+2 A))^{-1} \log \beta_{g, S}(k(R+2 A)) \\
& =\frac{R+2 A}{R} \varphi(g, S) .
\end{aligned}
$$

Thus, for all $R$ there is $S \subset \Gamma$ such that

$$
\frac{R}{R+2 A} h(g) \leq \varphi(g, S) \leq h(g),
$$

and letting $R \rightarrow \infty$ completes the proof. 
Remark 2. We have shown the geometrical relevance of the supremum of $\varphi(g, S)$ over generating sets $S$. By contrast, the uniform exponential growth rate is defined as the infimum of $\exp \psi(\Gamma, S)$ taken over finite sets $S$ that generate $\Gamma$; see [2, 5.11] or [4 1, 7]. This is relevant to the abstract group $\Gamma$ rather than to its geometrical properties.

\section{REFERENCES}

[1] L. Bartholdi, A Wilson group of non-uniformly exponential growth, C. R. Math. Acad. Sci. Paris 336 (2003), no. 7, 549-554. MR 1981466 (2004c:20051)

[2] M. Gromov, Metric structures for Riemannian and non-Riemannian spaces, Progress in Mathematics, 152. Birkhäuser Boston, Inc., Boston, MA, 1999. MR.1699320 (2000d:53065)

[3] P. de la Harpe, Topics in geometric group theory, Chicago Lectures in Mathematics. University of Chicago Press, Chicago, IL, 2000. MR,1786869 (2001i:20081)

[4] - Uniform growth in groups of exponential growth, Geom. Dedicata 95 (2002), 1-17. MR,1950882(2003k:20031)

[5] A. Katok and B. Hasselblatt, Introduction to the modern theory of dynamical systems, Encyclopedia of Mathematics and its Applications, 54. Cambridge University Press, Cambridge, 1995. MR 1326374 (96c:58055)

[6] A. Manning, Topological entropy for geodesic flows, Ann. of Math. (2), 110 (1979), no. 3, 567-573. MR0554385 (81e:58044)

[7] D. Osin, The entropy of solvable groups, Ergodic Theory Dynam. Systems 23 (2003), no. 3, 907-918. MR 1992670 (2004f:20065)

[8] G. Paternain, Geodesic flows, Progress in Mathematics, 180. Birkhäuser Boston, Inc., Boston, MA, 1999. MF 1712465 (2000h:53108)

Mathematics Institute, University of Warwick, Coventry, CV4 7AL, United Kingdom

E-mail address: akm@maths.warwick.ac.uk 